Imperial-TP-EM-2010-01

\title{
On nonlinear classical electrodynamics with an axionic term
}

\author{
E. M. Murchikova* \\ Blackett Laboratory, Imperial College London, SW7 $2 A Z$ London, UK \\ Skobeltsyn Institute of Nuclear Physics, Moscow State University \\ 119991 Moscow, Russia
}

\begin{abstract}
Recently there has been a renewed interest in axionic generalization of electrodynamics due to its application to topological insulators. A low-energy electromagnetic response of these exotic materials was proposed to be described by an axionic term in the Lagrangian. Motivated by this it is of interest to study various aspects of axionic electrodynamics and analyze the universal features of the axionic effects. Here we discuss the axionic modification of generalized electrodynamics with a Lagrangian being an arbitrary function of two electromagnetic invariants. Surprisingly, the qualitative characteristics of the major axionic effects known in the Maxwell theory happen to be independent of the exact type of the nonlinear Lagrangian and are uniquely fixed by the form of the axionic term.
\end{abstract}

Topological insulator (TI) is a recently discovered type of materials. An anomalous band structure makes it insulating in the bulk and an exotic metal on the surface and boundary (electrons on its surfaces are insensitive to scattering by impurities). The first reported example of such (2D) states is the quantum Hall effect, where "the metal" occurs on the edges of the sample. Similar behaviour was predicted to appear [1,2 and experimentally discovered [3 5] in several 3D materials: $\mathrm{Bi}_{1-\mathrm{x}} \mathrm{Sb}_{\mathrm{x}}$ alloys and $\mathrm{Bi}_{2} \mathrm{Se}_{3}, \mathrm{Bi}_{2} \mathrm{Te}_{3}$, where the role of the magnetic field is assumed by spin-orbital coupling and intrinsic properties of matter. Exotic characteristics make TI a promising candidate for applications in quantum computing, in particular for constructing a quantum bit (for a review on TI see [6]).

It was proposed, that a low-energy electromagnetic response of TI is described by the axionic term in the Lagrangian [3]

$$
L_{\theta}=\theta(\mathbf{E} \cdot \mathbf{B}) .
$$

Influence of this term on Maxwell electrodynamics was initially studied many years ago and several fascinating properties of an interface between two media with different values of $\theta$ (an interface with a non-trivial jump of $\theta$ ) were reported: magnetic charges induce electric mirror charges and vice-versa [7]; a magnetic monopole surrounded by a spherical axionic domain boundary picks up a non-trivial electric charge [8]; reflection off an axionic domain wall induces a non-trivial rotation of the polarization of the fields [9,10]. Some of these phenomena are expected to be experimentally observable at the surface of TI [11.

In light of the renewed interest in the axionic electrodynamics 1 , it is of interest to turn again to theoretical exploration of various aspects of axionic electrodynamics and analyze the common features of the axionic affects not related to particular type of electrodynamics chosen. To this end we discuss

\footnotetext{
*e-mail: e.murchikova@imperial.ac.uk

${ }^{1}$ It may be mentioned that the Lagrangian describing a real antiferromagnet chromium oxide $\mathrm{Cr}_{2} \mathrm{O}_{3}$ was also shown to have an axionic piece [12].
} 
axionic modification of the generalized electrodynamics, the theory with the Lagrangian which is an arbitrary function of two electromagnetic invariants. Surprisingly, the qualitative characteristics of the major axionic effects expected on the basis of Maxwell theory turn out to be uniquely fixed by the form of the axionic term and are independent on the exact form of the electrodynamics Lagrangian.

Before discussing the generalized theory we consider a particular example of nonlinear electrodynamics - the Born-Infeld (BI) theory [13] (section 2). We derive the laws of refraction for electric and magnetic fields at the interface with non-trivial jump of $\theta$ and make use of them when considering electrically and magnetically charged plane at the axionic boundary. Section 3 is dedicated to a discussion of quantitative and qualitative predictions of axionic effects in generalized electrodynamics with Lagrangian as an arbitrary function of two electromagnetic invariants. In Appendix A we consider an alternative approach to effective generalization of axionic electrodynamics at the level of equations of motion. Depending on the way of treating magnetic monopoles (as genuine or just an effective objects) there is an ambiguity in performing such a generalization. It results, for example, in opposite predictions for existence of the Witten effect in a specific configuration of magnetic charges and axionic fields.

\section{BI electrodynamics with an axionic term}

\subsection{Maxwell equations for the BI axionic electrodynamics}

We start with a detailed analysis of a particular example of the nonlinear electrodynamics — the Born-Infeld (BI) theory [13].

Similarly to the Maxwell theory, the BI axionic electrodynamic is classically invariant under the $S L(2, R)$ duality transformations [14-16] (see [17] for a discussion of this symmetry in the context of TIs in Maxwell theory). Originally it was formulated [13] as an extension of the standard Maxwell electromagnetism where self-energy of a point electric charge is finite. Interest in this theory was revived as it was shown that the BI-type Lagrangian appears in string theory (see [18] and references therein). In particular, the world volume action for a bosonic D3-brane is of BI type with the axionic term accounting for the coupling to a Ramond-Ramond scalar field background (see, e.g., [19]).

The Lagrangian of BI electrodynamics with the axionic term and a source reads

$$
\begin{aligned}
& L_{\mathrm{BI}+\theta} \\
& \quad=b^{2}\left(1-\sqrt{1+\frac{F^{\mu \nu} F_{\mu \nu}}{2 b^{2}}-\frac{\left({ }^{*} F^{\mu \nu} F_{\mu \nu}\right)^{2}}{16 b^{4}}}\right)+\frac{1}{4} \theta^{*} F^{\mu \nu} F_{\mu \nu}-A_{\mu} j_{\mathrm{e}}^{\mu} \\
& \quad=b^{2}\left(1-\sqrt{1+b^{-2}\left(\mathbf{B}^{2}-\mathbf{E}^{2}\right)-b^{-4}(\mathbf{E} \cdot \mathbf{B})^{2}}\right)+\theta(\mathbf{E} \cdot \mathbf{B})-A_{\mu} j_{\mathrm{e}}^{\mu} .
\end{aligned}
$$

Here $F_{\mu \nu}=\partial_{\mu} A_{\nu}-\partial_{\nu} A_{\mu},{ }^{*} F^{\mu \nu}=-\frac{1}{2} e^{\mu \nu \rho \lambda} F_{\rho \lambda}, e^{\mu \nu \rho \lambda}$ is the totaly antisymmetric tensor $\left(e^{0123}=1\right)$, $\frac{1}{4} F^{\mu \nu} F_{\mu \nu}=\frac{1}{2}\left(\mathbf{B}^{2}-\mathbf{E}^{2}\right), \frac{1}{4}{ }^{*} F^{\mu \nu} F_{\mu \nu}=(\mathbf{E} \cdot \mathbf{B}), j_{\mathrm{e}}^{\mu}=\left\{\rho_{\mathrm{e}}, \mathbf{j}_{\mathrm{e}}\right\}$ is an electric four-current and $b$ plays the role of electric field cut-off (if $\mathbf{B}=0$, then $|\mathbf{E}|<b$ ) 2 . When $\theta=\theta(x)$ is constant, the axionic term in (2) is a complete derivative:

$$
L_{\theta} \sim \theta \partial_{\mu}\left(e^{\nu \nu \rho \lambda} A_{\nu} \partial_{\rho} A_{\lambda}\right)
$$

and does not effect equations of motion. Further we will discuss varying $\theta(x)$.

\footnotetext{
${ }^{2}$ The value of $b$ is defined by $b=\frac{E_{\max }}{c}$, where $E_{\max }=\frac{e}{4 \pi \epsilon_{0} r_{0}^{2}}$ is the maximal field strength achieved in the Coulomb configuration, $r_{0} \approx r_{e}=\frac{e^{2}}{4 \pi \epsilon_{0} m c^{2}}$ is the classical radius of the electron. Explicitly $b \approx 6.4 \times 10^{11} \frac{\mathrm{V} \cdot \mathrm{sec}}{\mathrm{m}^{2}}$.
} 
From (2) and the Bianchi identity

$$
\partial_{\mu}^{*} F^{\mu \nu}=0
$$

we obtain the set of Maxwell equations

$$
\left\{\begin{array}{l}
\nabla \cdot \mathbf{D}=\rho_{\mathrm{e}} \\
\nabla \cdot \mathbf{B}=0 \\
-\nabla \times \mathbf{E}=\frac{\partial \mathbf{B}}{\partial t} \\
\nabla \times \mathbf{H}=\frac{\partial \mathbf{D}}{\partial t}+\mathbf{j}_{\mathrm{e}}
\end{array}\right.
$$

Here the electric displacement and magnetizing fields are defined respectively as

$$
\begin{aligned}
\mathbf{D} & =\frac{\partial L_{\mathrm{BI}+\theta}}{\partial \mathbf{E}} \mathbf{E}+b^{-2}(\mathbf{E} \cdot \mathbf{B}) \mathbf{B} \\
& =\frac{{ }^{1+b^{-2}\left(\mathbf{B}^{2}-\mathbf{E}^{2}\right)-b^{-4}(\mathbf{E} \cdot \mathbf{B})^{2}}}{\sqrt{L_{\mathrm{BI}+\theta}}}+\theta \mathbf{B}=\mathbf{D}_{0}+\theta \mathbf{B}, \\
& =-\frac{\partial \mathbf{B}}{\mathbf{B}-b^{-2}(\mathbf{E} \cdot \mathbf{B}) \mathbf{E}} \\
& =\frac{b^{1+b^{-2}\left(\mathbf{B}^{2}-\mathbf{E}^{2}\right)-b^{-4}(\mathbf{E} \cdot \mathbf{B})^{2}}}{\sqrt{1+}}=\mathbf{H}_{0}-\theta \mathbf{E},
\end{aligned}
$$

where the notations $\mathbf{D}_{0}$ and $\mathbf{H}_{0}$ stand for the fields in the non-axionic BI theory.

\subsection{Refraction of electric and magnetic fields at the axionic domain boundary}

Let us consider a planar axionic domain wall at $z=0$ :

$$
\theta(z)= \begin{cases}\theta_{1} & \text { for } z<0 \\ \theta_{2} & \text { for } z \geq 0\end{cases}
$$

Our aim is to find the laws of refraction for electric and magnetic fields at such boundary — an analogue of the Snell's laws for BI axionic theory. Similarly to the optical Snell's laws which give the value and the direction of electric and magnetic fields on the other side of the interface with different refractive indexes, "axionic Snell's laws" give the value and the direction of electric and magnetic fields on the other side of the interface with different $\theta$.

In the absence of surface currents and charges Maxwell equations (5) demand continuity for the perpendicular $(\perp)$ to the boundary components of $\mathbf{D}$ and $\mathbf{B}$ and the parallel $(\|)$ components of $\mathbf{H}$ and E :

$$
\begin{array}{ll}
D_{\perp}^{+}=D_{\perp}^{-}, & B_{\perp}^{+}=B_{\perp}^{-}, \\
H_{\|}^{+}=H_{\|}^{-}, & E_{\|}^{+}=E_{\|}^{-},
\end{array}
$$

where "-" and "+" refer to the fields behind $(z<0)$ and in front $(z>0)$ of axionic wall, respectively. 
In the explicit form (8) read

$$
\begin{aligned}
& B_{\perp}^{+}=B_{\perp}^{-}, \\
& E_{\|}^{+}=E_{\|}^{-}, \\
& \frac{E_{\perp}^{+}+b^{-2}\left(\mathbf{E}^{+} \cdot \mathbf{B}^{+}\right) B_{\perp}^{+}}{\sqrt{1+b^{-2}\left(\mathbf{B}^{+2}-\mathbf{E}^{+2}\right)-b^{-4}\left(\mathbf{E}^{+} \cdot \mathbf{B}^{+}\right)^{2}}}+\theta_{2} B_{\perp}^{+} \\
& \quad=\frac{E_{\perp}^{-}+b^{-2}\left(\mathbf{E}^{-} \cdot \mathbf{B}^{-}\right) B_{\perp}^{-}}{\sqrt{1+b^{-2}\left(\mathbf{B}^{-2}-\mathbf{E}^{-2}\right)-b^{-4}\left(\mathbf{E}^{-} \cdot \mathbf{B}^{-}\right)^{2}}}+\theta_{1} B_{\perp}^{-}, \\
& \frac{B_{\|}^{+}-b^{-2}\left(\mathbf{E}^{+} \cdot \mathbf{B}^{+}\right) E_{\|}^{+}}{\sqrt{1+b^{-2}\left(\mathbf{B}^{+2}-\mathbf{E}^{+2}\right)-b^{-4}\left(\mathbf{E}^{+} \cdot \mathbf{B}^{+}\right)^{2}}}-\theta_{2} E_{\|}^{+} \\
& =\frac{B_{\|}^{-}-b^{-2}\left(\mathbf{E}^{-} \cdot \mathbf{B}^{-}\right) E_{\|}^{-}}{\sqrt{1+b^{-2}\left(\mathbf{B}^{-2}-\mathbf{E}^{-2}\right)-b^{-4}\left(\mathbf{E}^{-} \cdot \mathbf{B}^{-}\right)^{2}}}-\theta_{1} E_{\|}^{-} .
\end{aligned}
$$

One may easily find the general solution of these equations. In particular cases it looks as follows: if $\mathbf{B}^{-} \neq 0, \quad \mathbf{E}^{-}=0$ :

$$
\begin{aligned}
& \mathbf{B}^{+}=\mathbf{B}^{-}, \quad E_{\|}^{+}=0, \\
& E_{\perp}^{+}=-\frac{\theta B_{\perp}^{-}}{\sqrt{1+b^{-2} B_{\perp}^{-2}\left(1+\theta^{2}\right)}} \sqrt{\frac{1+b^{-2} \mathbf{B}^{-2}}{1+b^{-2} B_{\perp}^{-}}} ;
\end{aligned}
$$

if $\mathbf{B}^{-}=0, \quad \mathbf{E}^{-} \neq 0$ :

$$
\begin{aligned}
& B_{\perp}^{+}=0, \quad \mathbf{E}^{+}=\mathbf{E}^{-}, \\
& B_{\|}^{+}=\frac{\theta E_{\|}^{-}}{\sqrt{1-b^{-2} E_{\|}^{-2}\left(1+\theta^{2}\right)}} \sqrt{\frac{1-b^{-2} \mathbf{E}^{-2}}{1-b^{-2} E_{\|}^{-2}}} ;
\end{aligned}
$$

if $B_{\perp}^{-}, E_{\perp}^{-} \neq 0, \quad B_{\|}^{-}, E_{\|}^{-}=0$ :

$$
\begin{aligned}
& B_{\perp}^{+}=B_{\perp}^{-}, \quad B_{\|}^{+}=0, \quad E_{\|}^{+}=E_{\|}^{-}, \\
& E_{\perp}^{+}=\frac{\frac{E_{\perp}^{-}}{\sqrt{1-b^{-2} E_{\perp}^{-2}}}-\frac{\theta B_{\perp}^{-}}{\sqrt{1+b^{-2} B_{\perp}^{-2}}}}{\sqrt{1+b^{-2}\left(\frac{E_{\perp}^{-}}{\sqrt{1-b^{-2} E_{\perp}^{-2}}}-\frac{\theta B_{\perp}^{-}}{\sqrt{1+b^{-2} B_{\perp}^{-2}}}\right)^{2}}} ;
\end{aligned}
$$

if $B_{\perp}^{-}, E_{\perp}^{-}=0, \quad B_{\|}^{-}, E_{\|}^{-} \neq 0$ :

$$
\begin{aligned}
& B_{\perp}^{+}=0, \quad E_{\perp}^{+}=0, \quad E_{\|}^{+}=E_{\|}^{-}, \\
& B_{\|}^{+}=\frac{\frac{B_{\|}^{-}}{\sqrt{1+b^{-2} B_{\|}^{-2}}}+\frac{\theta E_{\|}^{-}}{\sqrt{1-b^{-2} E_{\|}^{-2}}}}{\sqrt{1-b^{-2}\left(\frac{B_{\|}^{-}}{\sqrt{1+b^{-2} B_{\|}^{-2}}}+\frac{\theta E_{\|}^{-}}{\sqrt{1-b^{-2} E_{\|}^{-2}}}\right)^{2}}},
\end{aligned}
$$


where $\theta=\theta_{2}-\theta_{1}$.

As an example of application of the BI axionic Snell's laws, consider a uniformly electrically $\rho_{\mathrm{e}}$ and magnetically $\rho_{\mathrm{m}}$ charged plane parallel to the wall (7) and located at $z=d<0$. The electric and magnetic fields at $z<0$ side of the wall are

$$
\begin{aligned}
& B_{\perp}^{-}=\rho_{\mathrm{m}} / 2, \\
& E_{\perp}^{-}=\frac{\rho_{\mathrm{e}}}{\sqrt{4+b^{-2}\left(\rho_{\mathrm{m}}^{2}+\rho_{\mathrm{e}}^{2}\right)}} .
\end{aligned}
$$

Using (12) one finds the fields on the other $(z>0)$ side:

$$
\begin{aligned}
& B_{\perp}^{+}=\rho_{\mathrm{m}} / 2, \\
& E_{\perp}^{+}=\frac{\rho_{\mathrm{e}}-\theta \rho_{\mathrm{m}}}{\sqrt{4+b^{-2}\left(\rho_{\mathrm{m}}^{2}+\left(\rho_{\mathrm{e}}-\theta \rho_{\mathrm{m}}\right)^{2}\right)}}
\end{aligned}
$$

We see that due to the axionic wall "shielding" the plane effectively picks up an additional electric charge density $\rho_{\mathrm{e}}^{\text {add }}=-\theta \rho_{\mathrm{m}}$, similarly to what is predicted in the framework of the Maxwell theory.

Axionic Snell's laws (10)-(13) are quite useful in calculation of various phenomena at an axionic boundary, however in most cases this approach is unnecessarily complicated. In the next section we will use an easier and more general approach.

\section{Generalized non-linear electrodynamics with the axionic term}

\subsection{Maxwell equations for the generalized electrodynamics}

Let us consider a generalized electrodynamics [21] with the axionic term and a source

$$
L_{\mathrm{gen}+\theta}=L_{\mathrm{gen}}+\frac{1}{4} \theta^{*} F^{\mu \nu} F_{\mu \nu}-A_{\mu} j_{\mathrm{e}}^{\mu},
$$

where $L_{\text {gen }}$ is an arbitrary electrodynamics Lagrangian, as a function of two electromagnetic invariant:3. 3 .

Maxwell equations obtained from (16) have the same form as (5) with the constitutive relations

$$
\begin{aligned}
& \mathbf{D}=\frac{\partial L_{\mathrm{gen}+\theta}}{\partial \mathbf{E}}=\frac{\partial L_{\mathrm{gen}}}{\partial \mathbf{E}}+\theta \mathbf{B}=\mathbf{D}_{0}+\theta \mathbf{B} \\
& \mathbf{H}=-\frac{\partial L_{\mathrm{gen}+\theta}}{\partial \mathbf{B}}=-\frac{\partial L_{\mathrm{gen}}}{\partial \mathbf{B}}-\theta \mathbf{E}=\mathbf{H}_{0}-\theta \mathbf{E} .
\end{aligned}
$$

Here $\mathbf{D}_{0}$ and $\mathbf{H}_{0}$ are the electric displacement and magnetizing fields in the $L_{\text {gen }}$ non-axionic theory. Using these notation one may rewrite (5) as follows

$$
\left\{\begin{array}{l}
\nabla \cdot \mathbf{D}_{0}=\rho_{\mathrm{e}}-\nabla \theta \cdot \mathbf{B} \\
\nabla \cdot \mathbf{B}=0 \\
\nabla \times \mathbf{E}=-\frac{\partial \mathbf{B}}{\partial t} \\
\nabla \times \mathbf{H}_{0}=\frac{\partial \mathbf{D}_{0}}{\partial t}+\mathbf{j}_{\mathrm{e}}+\mathbf{B} \frac{\partial}{\partial t} \theta+\nabla \theta \times \mathbf{E}
\end{array}\right.
$$

\footnotetext{
${ }^{3}$ Note, the function $L_{g e n}$ is not completely random, as it is assumed to be the Lagrangian of a consistent electrodynamics.
} 
Indeed, the primary objects in electrodynamics are $\mathbf{D}$ and $\mathbf{H}$ (not $\mathbf{D}_{0}, \mathbf{H}_{0}$ ), so e.g. an electric charge must be treated as a source of $\mathbf{D}$ field with $\theta \mathbf{B}$ as an inseparable part. However, so far as we treat magnetic charges as an effective objects and $\nabla \cdot \mathbf{B}=0$ is still applicable, only an electric charge is a source of an electric field and one may reinterpret axionic terms simply as additional charge and current densities 4 (see Appendix A for a discussion of an alternative approach to effective generalization of the Maxwell equations, when magnetic charges are treated as genuine physical objects and the use of $\nabla \cdot \mathbf{B}=0$ is not allowed).

\subsection{Uniformly charged plane}

Consider a uniformly $\rho_{\mathrm{e}}$ and $\rho_{\mathrm{m}}$ charged plane located at $z=d<0$ at the axionic wall (7):

$$
\left\{\begin{array}{l}
\nabla \cdot D_{0}=\rho_{\mathrm{e}} \delta(z+d)-\theta \delta(0) B(0) \\
\nabla \cdot B=\rho_{\mathrm{m}} \delta(z+d)
\end{array}\right.
$$

From this equations one finds that

$$
\left\{\begin{array}{l}
B^{+}=\rho_{\mathrm{m}} / 2 \\
D_{0}^{+}=D_{0}^{-}-\theta \rho_{\mathrm{m}}
\end{array}\right.
$$

These fields may be interpreted (see (18)) as the charged plane effectively receives an additional electric charge $-\theta \rho_{\mathrm{m}} / 2$. It is exactly the same result as we obtained in the previous section in the particular case of BI electrodynamics using the axionic Snell's laws. Here we generalized it to an arbitrary linear by source axionic electrodynamics.

\subsection{Spherical axionic boundary}

Consider a magnetic charge $q_{\mathrm{m}}$ surrounded by spherical axionic domain boundary of radius $r_{0}$ []:

$$
\theta(r)= \begin{cases}0 & \text { for } r<r_{0} \\ \theta & \text { for } r \geq r_{0}\end{cases}
$$

Integration of equations (18) over $0 \leq r \leq R, R>r_{0}$, using spherical symmetry of the problem, gives

$$
D_{0}=-\frac{\theta q_{\mathrm{m}}}{R^{2}}
$$

We see that a magnetic charge in this configuration of $\theta(r)$, effectively picks up an electric charge $q_{\mathrm{e}}=-\theta q_{\mathrm{m}}$ and become a dyon. This result is true in an arbitrary electrodynamics.

\subsection{Time-dependent $\theta$}

Consider linearly time-dependent $\theta[20]$ :

$$
\theta(x)=\theta_{0} t
$$

and a magnetic charge $\rho_{\mathrm{m}}=q_{\mathrm{m}} \delta(\mathbf{r})$ :

$$
\left\{\begin{array}{l}
\nabla \cdot \mathbf{D}_{0}=\rho_{\mathrm{e}} \\
\nabla \cdot \mathbf{B}=q_{\mathrm{m}} \delta(\mathbf{r}) \\
\nabla \times \mathbf{E}=-\frac{\partial \mathbf{B}}{\partial t} \\
\nabla \times \mathbf{H}_{0}=\frac{\partial \mathbf{D}_{0}}{\partial t}+\mathbf{B} \frac{\partial}{\partial t} \theta(x)
\end{array}\right.
$$

\footnotetext{
${ }^{4}$ Note, that at an axionic boundary constrains (8) hold for $\mathbf{D}, \mathbf{H}$, while $\mathbf{D}_{0}$ and $\mathbf{H}_{0}$ experience jumps.
} 
Taking the divergence of the fourth equation in (24), one finds

$$
\frac{\partial}{\partial t} \rho_{\mathrm{e}}=-\theta_{0} \nabla \cdot \mathbf{B}=-\theta_{0} q_{\mathrm{m}} \delta(\mathbf{r})
$$

Thus a magnetic charge in linearly increasing/decreasing with time axionic background (23) become a dyon linearly increasing/decreasing its electric charge.

\subsection{Comments on mirror charges}

All axionic effects are strongly restricted by two constrains following directly from Maxwell equations (18). Firstly, all axionic effects are related to $B_{\perp}$ and $E_{\|}$. Secondly, the continuity relations require $B_{\perp}^{-}=B_{\perp}^{+}$and $E_{\|}^{-}=E_{\|}^{+}$at the axionic boundary, which is free of charges and currents. In these bounds axionic Snell's laws of any axionic electrodynamics would be qualitatively the same. Thus the mirror charge effects must be also very similar to ones in the Maxwell case, i.e. electric charges would induce magnetic mirror charges and vice-versa.

However, one may not demand the mirror charges to be point-like. In particular, in BI electrodynamics an electric charge is point-like in terms of $\mathbf{D}_{0}$, but a charge density in terms of $\mathbf{E}$. In this context, magnetic charges are expected to be represented by charge densities, as the axionic term establishes relation between $\mathbf{B}$ and $\mathbf{E}$.

\section{Conclusion}

In this paper we have demonstrated that nonlinear modifications of the ordinary Maxwell electrodynamics does not change the qualitative, and in some cases also the quantitative, predictions of the influence of the axionic term.

\section{Acknowledgments}

I would like to thank Arkady Tseytlin for many helpful discussions and careful reading of the draft of this manuscript and Yuri Obukhov for comments and criticism. This work is supported by a grant of Dynasty Foundation and in part by a grant of President of the Russian Federation for Leading Scientific Schools (Grant No. SS-4142.2010.2).

\section{A Axionic electrodynamics with magnetic monopoles. Effective generalization at the level of equations of motion}

In this section we start from equations of motion for an arbitrary axionic electrodynamics (5) and, following Dirac, generalize Bianchi identity (4) to include into consideration a magnetic four-current $j_{\mathrm{m}}^{\nu}=\left\{\rho_{\mathrm{m}}, \mathbf{j}_{\mathrm{m}}\right\}$ :

$$
\partial_{\mu}{ }^{*} F^{\mu \nu}=j_{\mathrm{m}}^{\nu}
$$

In this case the generalized Maxwell equations read

$$
\left\{\begin{array}{l}
\nabla \cdot\left(\mathbf{D}_{0}+\theta \mathbf{B}\right)=\rho_{\mathrm{e}} \\
\nabla \cdot \mathbf{B}=\rho_{\mathrm{m}} \\
-\nabla \times \mathbf{E}=\frac{\partial \mathbf{B}}{\partial t}+\mathbf{j}_{\mathrm{m}} \\
\nabla \times\left(\mathbf{H}_{0}-\theta \mathbf{E}\right)=\frac{\partial}{\partial t}\left(\mathbf{D}_{0}+\theta \mathbf{B}\right)+\mathbf{j}_{\mathrm{e}}
\end{array}\right.
$$


where the constitutive relations are given by (17) with $\mathbf{D}_{0}$ and $\mathbf{H}_{0}$ being the electric displacement and magnetizing fields in the generalized non-axionic theory. Here we treat magnetic monopoles as genuine physical objects, thus the use of $\nabla \cdot \mathbf{B}=0$ is not allowed.

A shift of $\theta$ by a constant no longer leaves Maxwell equations (27) invariant. We may question how the electrodynamics with the additional axionic term ( $\theta$-electrodynamics) is different from the non-axionic one.

Consider a pure electric charge density $\rho_{\mathrm{e}}$ in a space with non-trivial $\theta$ :

$$
\left\{\begin{array}{l}
\nabla \cdot\left(\mathbf{D}_{0}+\theta \mathbf{B}\right)=\rho_{\mathrm{e}} \\
\nabla \cdot \mathbf{B}=\rho_{\mathrm{m}}=0
\end{array}\right.
$$

From these equations one finds that the electric charge density is a source of the same field configuration as in the non-axionic theory:

$$
\left\{\begin{array}{l}
\nabla \cdot \mathbf{D}_{0}=\rho_{\mathrm{e}} \\
\nabla \cdot \mathbf{B}=0
\end{array}\right.
$$

Now consider a pure magnetic charge density $\rho_{\mathrm{m}}$ :

$$
\left\{\begin{array}{l}
\nabla \cdot\left(\mathbf{D}_{0}+\theta \mathbf{B}\right)=\rho_{\mathrm{e}}=0 \\
\nabla \cdot \mathbf{B}=\rho_{\mathrm{m}}
\end{array}\right.
$$

From these equations one finds

$$
\left\{\begin{array}{l}
\nabla \cdot \mathbf{D}_{0}=-\theta \rho_{\mathrm{m}} \\
\nabla \cdot \mathbf{B}=\rho_{\mathrm{m}}
\end{array}\right.
$$

The magnetic charge density generates a field configuration, which is equivalent to the one generated by a dyon in the non-axionic electrodynamics, in other words $\rho_{\mathrm{m}}$ effectively induces an electric charge density $\rho_{\mathrm{e}}^{\mathrm{eff}}=-\theta \rho_{\mathrm{m}}$.

Though non-trivial $\mathbf{D}_{0}$, resulting from $\rho_{\mathrm{m}}$, is just an effective response of the axionic medium, a probe electric charge will interact with $\rho_{\mathrm{m}}$ via electric force. The latter, in fact, runs us into a problem: the object called "a magnetic charge" may not statically interact with an electric charge. In this sense, for an observer located in a space with non-trivial $\theta$ (a $\theta$-observer), $\rho_{\mathrm{m}}$ is not a magnetic, but a dyonic charge density.

Concerning electric and magnetic currents, one reaches similar conclusions:

$$
\left\{\begin{array} { l } 
{ - \nabla \times \mathbf { E } = 0 } \\
{ \nabla \times ( \mathbf { H } _ { 0 } - \theta \mathbf { E } ) = \mathbf { j } _ { \mathrm { e } } }
\end{array} \Rightarrow \left\{\begin{array}{l}
-\nabla \times \mathbf{E}=0 \\
\nabla \times \mathbf{H}_{0}=\mathbf{j}_{\mathrm{e}}
\end{array}\right.\right.
$$

and

$$
\left\{\begin{array} { l } 
{ - \nabla \times \mathbf { E } = \mathbf { j } _ { \mathrm { m } } } \\
{ \nabla \times ( \mathbf { H } _ { 0 } - \theta \mathbf { E } ) = 0 }
\end{array} \Rightarrow \left\{\begin{array}{l}
-\nabla \times \mathbf{E}=\mathbf{j}_{\mathrm{m}} \\
\nabla \times \mathbf{H}_{0}=-\theta \mathbf{j}_{\mathrm{m}}
\end{array}\right.\right.
$$

While the electric current is a source of the same fields as in the non-axionic theory, the magnetic current $\mathbf{j}_{\mathrm{m}}$ generates the field configuration equivalent to one generated by a dyonic current with $\mathbf{j}_{\mathrm{e}}^{\mathrm{eff}}=-\theta \mathbf{j}_{\mathrm{m}}$.

Let us rewrite Maxwell equations (27) in terms of new four-currents $\tilde{j}_{\mathrm{e}, \mathrm{m}}$, which are the pure sources of electric and magnetic fields in $\theta$ - electrodynamics 5 :

$$
\begin{aligned}
& \tilde{j}_{\mathrm{e}}^{\mu} \equiv\left\{j_{\mathrm{e}}^{\mu}, j_{\mathrm{m}}^{\mu}=0\right\} \\
& \tilde{j}_{\mathrm{m}}^{\mu} \equiv\left\{j_{\mathrm{e}}^{\mu}=\theta j_{\mathrm{m}}^{\mu}, j_{\mathrm{m}}^{\mu}\right\} .
\end{aligned}
$$

\footnotetext{
${ }^{5}$ See [17] for the considerations of the same transformations in explicitly $S L(2, R)$ duality covariant form in the case of Maxwell electrodynamics
} 
The resulting equations are the same as in the non-axionic electrodynamics:

$$
\left\{\begin{array}{l}
\nabla \cdot \mathbf{D}_{0}=\tilde{\rho}_{\mathrm{e}} \\
\nabla \cdot \mathbf{B}=\tilde{\rho}_{\mathrm{m}} \\
-\nabla \times \mathbf{E}=\frac{\partial \mathbf{B}}{\partial t}+\tilde{\mathbf{j}}_{\mathrm{m}} \\
\nabla \times \mathbf{H}_{0}=\frac{\partial \mathbf{D}_{0}}{\partial t}+\tilde{\mathbf{j}}_{\mathrm{e}}
\end{array}\right.
$$

Non-axionic and $\theta$ - electrodynamics are dynamically indistinguishable, but have different definitions of a magnetic four-current. Globally it means that, when $\theta$ is varying in space, the differentiation of "magnetic" or "dyonic" four-currents is also varying in space with respect to the value of $\theta$ at the concrete position of an observer].

Discuss now a magnetic charge $Q_{\mathrm{m}}$ surrounded by a spherical vacuum bubble of radius $r_{0}$ (21) [8]. To start, one must clarify the meaning of the magnetic charge $Q_{\mathrm{m}}$. It must be approved to be a magnetic charge by an observer located at $r>r_{0}$ (a $\theta$-observer), who is actually doing this experiment. The object defined as a monopole in $\theta$-system is a dyon

$$
Q_{\mathrm{m}}=\left\{q_{\mathrm{e}}=\theta q_{\mathrm{m}}, q_{\mathrm{m}}\right\},
$$

where $q_{\mathrm{e}}, q_{\mathrm{m}}$ are non-axionic electric and magnetic charges. Inserting (36) into the bubble, one finds

$$
\begin{aligned}
& D^{\text {in }}=\theta \frac{q_{m}}{r^{2}} \\
& B^{\text {in }}=\frac{q_{m}}{r^{2}}
\end{aligned} \quad \Rightarrow \quad \begin{aligned}
& D^{\text {out }}=D_{0}^{\text {out }}+\theta B^{\text {out }}=\theta \frac{q_{m}}{r^{2}} \quad \Rightarrow D_{0}^{\text {out }}=0 . \\
& B^{\text {out }}=\frac{q_{m}}{r^{2}}
\end{aligned}
$$

Here in and out are refereed to the fields inside and outside the spherical axionic boundary, respectively.

The magnetic charge does not pick up an electric charge. This conclusion is opposite to one made in [8]. The reason is the definition of a monopole. The object that were put inside the bubble in [8] is $q_{\mathrm{m}}$, which is a magnetic monopole in non-axionic electrodynamics, but a dyon $\left\{-\theta Q_{\mathrm{m}}, Q_{\mathrm{m}}\right\}$ for a true $\theta$-observer. Thus the observer sees exactly the same dyon as were put inside the bubble in the beginning. The latter practically means that, in this approach, there is no Witten effect in this configuration.

\section{References}

[1] L. Fu and C. L. Kane, "Topological insulators with inversion symmetry," Phys. Rev. B 76, 045302 (2007).

[2] H. Zhang, C.-X. Liu, X.-L. Qi, X. Dai, Z. Fang and S.-C. Zhang, "Topological insulators in $\mathrm{Bi}_{2} \mathrm{Se}_{3}, \mathrm{Bi}_{2} \mathrm{Te}_{3}$ and $\mathrm{Sb}_{2} \mathrm{Te}_{3}$ with a single Dirac cone on the surface," Nature Phys. 5, 438 (2009).

[3] X.-L. Qi, T. Hughes and S.-C. Zhang, "Topological Field Theory of Time-Reversal Invariant Insulators," Phys. Rev. B 78, 195424 (2008) arXiv:0802.3537 [cond-mat.mes-hall].

\footnotetext{
${ }^{6}$ One must be extremely carefully defining magnetic four-current in each particular problem and always refer to the location of the observer. To avoid necessity of working with redefined magnetic four-current, one may always shift $\theta(x)$ in a way to put an observer into $\theta=0$ part of space, where the definition takes the conventional form.
} 
[4] Y. Xia et al., "Observation of a large-gap topological-insulator class with a single Dirac cone on the surface," Nature Phys. 5, 398 (2009).

[5] Y. L. Chen et al., "Experimental realization of a three-dimentional topological insulator, $\mathrm{Bi}_{2} \mathrm{Te}_{3}$," Science 325, 178 (2009).

[6] J. E. Moore, "The birth of topological insulators," Nature 464, 194 (2010).

[7] P. Sikivie, "On The Interaction Of Magnetic Monopoles With Axionic Domain Walls," Phys. Lett. B 137, 353 (1984).

[8] F. Wilczek, "Two applications of axion electrodynamics," Phys. Rev. Lett. 58, 1799 (1987).

[9] M. C. Huang and P. Sikivie, "The Structure Of Axionic Domain Walls," Phys. Rev. D 32, 1560 (1985).

[10] Y. N. Obukhov and F. W. Hehl, "Measuring a piecewise constant axion field in classical electrodynamics," Phys. Lett. A 341, 357 (2005) arXiv:physics/0504172.

[11] X.-L. Qi, R. Li, J. Zang and S.-C. Zhang, "Inducing a Magnetic Monopole with Topological Surface States," Science 323, 1184 (2009).

[12] F. W. Hehl, Yu. N. Obukhov, J.-P. Rivera and H. Schmid, "Relativistic nature of a magnetoelectric modulus of $\mathrm{Cr}_{2} \mathrm{O}_{3}$ crystals: A four-dimensional pseudoscalar and its measurement," Phys. Rev. A 77022106 (2008).

[13] M. Born and L. Infeld, "Foundations Of The New Field Theory," Proc. Roy. Soc. Lond. A 144, 425 (1934).

[14] G. W. Gibbons and D. A. Rasheed, "Electric - magnetic duality rotations in nonlinear electrodynamics," Nucl. Phys. B 454, 185 (1995) arXiv:hep-th/9506035.

[15] G. W. Gibbons and D. A. Rasheed, "SL(2,R) Invariance of Non-Linear Electrodynamics Coupled to An Axion and a Dilaton," Phys. Lett. B 365, 46 (1996) arXiv:hep-th/9509141.

[16] A. A. Tseytlin, "Self-duality of Born-Infeld action and Dirichlet 3-brane of type IIB superstring theory," Nucl. Phys. B 469, 51 (1996) arXiv:hep-th/9602064.

[17] A. Karch, "Electric-Magnetic Duality and Topological Insulators," Phys. Rev. Lett. 103, 171601 (2009) arXiv:0907.1528 [cond-mat.mes-hall]].

[18] A. A. Tseytlin, "Born-Infeld action, supersymmetry and string theory," arXiv:hep-th/9908105.

[19] C. V. Johnson, "D-Branes," Cambridge, USA: Univ. Pr. (2003) 548 p

[20] G. Rosenberg and M. Franz, "Witten effect in a crystalline topological insulator," arXiv:1001.3179 [cond-mat.mes-hall].

[21] J. Plebanski "Lectures on Non-Linear Electrodynamics", Nordita Lecture Notes (1968) 